\title{
Reprodução Induzida da Pirapitinga-do-Sul, Brycon opalinus (Cuvier, 1819), Mantida em Condições de Confinamento*
}

\section{Massuka Yamane Narahara1, Elaine Fender de Andrade-Talmelli ${ }^{1}$, Emico Tahira Kavamoto ${ }^{1}$, Heloisa Maria Godinho1}

RESUMO - Este estudo foi conduzido com o objetivo de obter a reprodução da pirapitinga-do-sul, Brycon opalinus, por intermédio da aplicação de hormônios indutores e determinar a distribuição de freqüência dos diâmetros dos ovócitos em fêmeas aptas à indução. Cento e vinte reprodutores apresentando comprimento entre 25,5 e 32,0 cm foram mantidos em um tanque circular de alvenaria, de $10 \mathrm{~m}$ de diâmetro, na Estação de Aqüicultura de Paraibuna/CESP. Para determinação das fêmeas aptas à reprodução, retiraram-se amostras de ovócitos com cateter plástico introduzido pela abertura urogenital. Estes ovócitos foram analisados quanto à cor, tamanho, posição da vesícula germinativa (núcleo) e distribuição de freqüência porcentual dos diâmetros. Os machos foram selecionados pela qualidade do sêmen. Vinte e nove fêmeas aptas à reprodução receberam duas injeções intramusculares de EPS (extrato de pituitária de salmão), sendo a $1^{\mathrm{a}}$, na dose de $5 \mathrm{mg} / \mathrm{kg}$ e a $2^{\mathrm{a}}$, de $10 \mathrm{mg} / \mathrm{kg}$, com intervalo de 12 horas. Os machos que apresentavam ao redor de $90 \%$ de motilidade espermática e $85 \%$ de espermatozóides vivos receberam dose única de 5 UI de hCG/g (gonadotropina coriônica humana), por ocasião

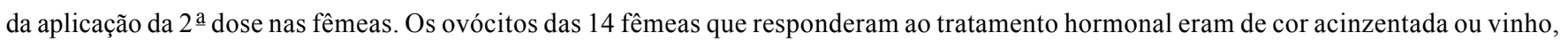
e aquelas que apresentaram alta taxa de fertilização mostraram distribuição de freqüência porcentual dos diâmetros com moda igual a $1900 \mu \mathrm{m}$. A taxa média de fertilização foi de $90 \%$, e a de eclosão, $40 \%$.

Palavras-chave: peixe, Brycon opalinus, pirapitinga-do-sul, reprodução induzida

\section{Induced Spawning of Pirapitinga-do-Sul, Brycon opalinus, in Captivity Conditions}

ABSTRACT - The objective of this study was to induce spawning in pirapitinga-do-sul, Brycon opalinus, with the application of hormones and to determine the pattern of oocyte diameter frequency distribution in females prepared to induction. One hundred and twenty spawners with 25.5 to $32.0 \mathrm{~cm}$ of length were placed in circular ponds of $10 \mathrm{~m}$ of diameter, at Estação de Aqüicultura de ParaibunaSP of CESP. In order to determine the females apt to reproduction, samples of oocytes were obtained with plastic catheter and analysed. These oocytes were valued by colour, size, position of nucleus and oocyte diameter percentual frequency distribution. The males were selected by quality of semen. Twenty-nine females were selected and received two injections of SPE (salmon pituitary extract): the first, of $5 \mathrm{mg} / \mathrm{kg}$ and the other, of $10 \mathrm{mg} / \mathrm{kg}$, with 12 hours of interval. The males that showed $90 \%$ of spermatic motility and $85 \%$ of life spermatozoa were selected and received $5 \mathrm{IU} / \mathrm{g}$ of hCG (human chorionic gonadotropin) when the females received the second dose. The oocytes of the 14 females that responded to the hormone treatment were gray or wine in colour, and in the females that presented high rates of fertilization, the oocyte diameter percentual frequency distribution showed mode of 1,900 mm. The average rate of fertilization was of $90 \%$ and that of hatching was $40 \%$.

Key Words: fish, Brycon opalinus, pirapitinga-do-sul, induced spawning

\section{Introdução}

A pirapitinga-do-sul, Brycon opalinus, pertencente à família Characidae, é um peixe de porte médio, muito apreciado pelos habitantes das regiões próximas à sua área de distribuição, ou seja, a Bacia do Rio Paraíba do Sul.

A população dessa espécie vem sofrendo declínio, principalmente em rios do Vale do Paraíba, um de seus hábitats naturais, em conseqüência de fatores que interferem no comportamento migratório das espécies ictíicas, dentre eles, o desmatamento marginal dos rios, a poluição e a construção de barragens hidrelétricas. Diante deste fato, programas de povoamento e de repovoamento de rios e de reservatórios tornam-se necessários (Torloni, 1992).

À semelhança de grande número de espécies reofílicas, que não se reproduzem naturalmente em condições de cativeiro, exemplares de Brycon opalinus necessitam ser induzidos à reprodução atra-

\footnotetext{
* Convênio CESP (Companhia Energética de São Paulo) e IP/SAA (Instituto de Pesca da Secretaria de Agricultura e Abastecimento do Estado de São Paulo)

${ }^{1}$ Pesquisador Científico - Centro de Pesquisa em Reprodução e Larvicultura - Instituto de Pesca/SAA. Endereço: Av. Francisco Matarazzo, 455 - Água Branca- São Paulo-CEP-05001-900. E.mail: mnarahara@ig.com.br; etalmelli@ig.com.br
} 
vés de aplicação de hormônios (Narahara et al., 1994). Vários pesquisadores brasileiros têm-se interessado pela reprodução induzida de espécies reofílicas. Assim, foram desenvolvidos trabalhos com mandi, Pimelodus maculatus (Fenerich et al., 1974), dourado, Salminus maxillosus (DumontNeto et al., 1997), curimbatá, Prochilodus scrofa (Castagnolli \& Cyrino, 1980; Fenerich-Verani et al., 1984; Godinho et al., 1984b), curimatã-pacu, Prochilodus margravii (Sato et al., 1996a), curimatã-pioa, Prochilodus affinis (Sato et al., 1996b), pacu, Piaractus mesopotamicus (=Colossoma mitrei) (Godinho et al., 1977; 1982; Castagnolli \& Donaldson, 1981; Romagosa et al., 1985a, b; Godinho \& Godinho, 1986; Bernardino et al., 1987; Romagosa et al., 1990), que são espécies de alto valor comercial e indicadas para piscicultura.

Relativamente a espécies do gênero Brycon, alguns trabalhos de reprodução induzida foram recentemente realizados no Brasil com matrinxã, Brycon cephalus (Bernardino et al., 1993; Romagosa et al., 1994), com piracanjuba, Brycon orbignyanus (Belmont, 1994), com piabanha, Brycon insignis (Faria, 1994; Andrade-Talmelli, 1997), com Brycon lundii (Sato et al., 1997, entre outros).

Uma questão, que tem merecido atenção em trabalhos de reprodução induzida de peixes, é o critério para identificação de peixes aptos à indução. Fenerich-Verani et al. (1984) utilizaram, pela primeira vez, a distribuição da freqüência porcentual dos diâmetros dos ovócitos intraováricos como indicador do grau de desenvolvimento gonadal de fêmeas e verificaram que essa distribuição, nos exemplares de Prochilodus scrofa que se reproduziram, era do tipo unimodal, com moda ao redor de $1100 \mu \mathrm{m}$. Da mesma forma, distribuição unimodal foi registrada por Romagosa et al. (1990) em Piaractus mesopotamicus e por e Andrade-Talmelli (1997) em Brycon insignis. Já para matrinxã, Brycon cephalus, Romagosa (1998) verificou que fêmeas apresentando alta taxa de fertilização mostravam distribuição de freqüência porcentual dos diâmetros dos ovócitos com duas modas: 939 e $1001 \mu \mathrm{m}$.

Ainda, para selecionar fêmeas reprodutoras utiliza-se a posição do núcleo nos ovócitos como indicador do grau de desenvolvimento gonadal. Segundo Lemanova \& Sakun (1975), ovócitos em final de maturação apresentam o núcleo situado na periferia da célula, contrariamente ao que ocorre em ovócitos imaturos ou em maturação, em que a posição do núcleo é central.

Com relação à reprodução induzida de espécies do gênero Brycon, Eckmann (1984) conseguiu induzir à desova quatro exemplares de Brycon of erythropterus, utilizando extrato de pituitária de Abramis brama, porém não descreveu as características desses reprodutores que responderam positivamente ao tratamento hormonal.

Este trabalho foi desenvolvido com os objetivos de testar método de indução da reprodução em Brycon opalinus e determinar a distribuição dos valores dos diâmetros dos ovócitos das fêmeas aptas à indução.

\section{Material e Métodos}

Para a realização deste estudo foram examinados 120 reprodutores de pirapitinga-do-sul, Brycon opalinus, com três anos e quatro meses de idade, mantidos em condições de confinamento na Estação de Aqüicultura de Paraibuna, da Companhia Energética de São Paulo (CESP). Estes exemplares estiveram estocados em tanques circulares de 10 metros de diâmetro, na proporção de 1 peixe $/ \mathrm{m}^{2}$, e alimentados diariamente com ração comercial balanceada com $30 \%$ de PB, duas vezes ao dia, correspondendo a $1 \%$ do peso vivo nos meses de inverno e a 3\% nos demais meses.

Para verificar o estádio de desenvolvimento gonadal das fêmeas, uma amostra de ovócitos foi retirada com auxílio de cateter plástico introduzido no ovário pela abertura urogenital. Uma parte dessa amostra foi fixada em solução de Gilson (Simpson, 1951), por 30 minutos. Após este período, os ovócitos foram medidos sob microscópio estereoscópico ZEISS (obj. 2x), com auxílio de ocular micrométrica (10x). As freqüências porcentuais dos diâmetros dos ovócitos foram calculadas, por classe de $50 \mu \mathrm{m}$, e seus valores lançados em gráfico. A outra parte da amostra foi fixada em líquido de Serra $(60 \mathrm{~mL}$ de álcool $90 \% ; 30 \mathrm{~mL}$ de formalina; $10 \mathrm{~mL}$ de ácido acético glacial Lemanova \& Sakun, 1975) por um período ao redor de 1 minuto e, em seguida, analisada sob estereomicroscópio para verificação da posição da vesícula germinativa (núcleo).

O estádio de desenvolvimento gonadal dos machos foi avaliado através de amostra de sêmen coletada por massagem realizada no abdome, no sentido 
ântero-posterior. O material seminal foi analisado quanto aos aspectos macroscópicos: cor, fluidez, viscosidade, e microscópicos: motilidade direta subjetiva (sobre uma lâmina, uma gota de sêmen misturada a duas gotas de solução de bicarbonato de sódio $1 \%$ e analisada sob microscópio de contraste de fase 400x), coloração diferencial para determinação da proporção de espermatozóides vivos e mortos (método de Blom, 1950 - avaliação feita através de contagem de células espermáticas móveis no campo microscópico focalizado, e determinando-se a porcentagem seguindo a escala arbitrária de 0 a $100 \%$ ) e concentração de espermatozóides $\left(\mathrm{n} / \mathrm{mm}^{3}\right)$ (determinada por contagem em câmara hematimétrica de Neubauer, "Improved").

Dos reprodutores selecionados de acordo com critério utilizado por Fenerich-Verani et al. (1984), anotaram-se dados de comprimento total, em centímetro, e peso total, em grama. Formaram-se 29 grupos, sendo cada grupo constituído por uma fêmea e dois machos e colocado em aquário com 1000 litros de água, aeração constante e temperatura de $21 \pm 1^{\circ} \mathrm{C}$.

As fêmeas selecionadas receberam duas injeções intramusculares de EPS (extrato de pituitária de salmão), nas doses de 5 e $10 \mathrm{mg} / \mathrm{kg}$, com intervalo de 12 horas. Os machos receberam aplicação de 5 UI de hCG/g (gonadotropina coriônica humana/grama) por ocasião da aplicação da segunda dose nas fêmeas.

Foram formados nove grupos controle que receberam duas injeções de solução salina a $0,9 \%$, com o mesmo espaço de tempo utilizado nos tratamentos hormonais.

Quando a desova não ocorreu naturalmente nos aquários, até 12 horas após a segunda aplicação hormonal, realizou-se a extrusão dos óvulos e fertilização pelo método a seco (Von Ihering \& Azevedo, 1934). Os ovos produzidos, por fêmea, foram colocados em incubadoras cilíndricas individuais contendo $20 \mathrm{~L}$ de água, corrente e à temperatura de $21 \pm 1^{\circ} \mathrm{C}$. Para estimar a taxa de fertilização, os ovos foram contados em duas subamostras de um litro e aplicouse a seguinte equação: Taxa de fertilização = número de ovos em divisão x 100/ número total de ovos. Para o cálculo da taxa de eclosão (Te), utilizaram-se duas subamostras de um litro cada uma, retiradas das incubadoras em que a eclosão das larvas já havia ocorrido, e aplicou-se a seguinte equação: $\mathrm{Te}=$ número de larvas eclodidas x 100/ número total de ovos.

\section{Resultados e Discussão}

As fêmeas de Brycon opalinus utilizadas neste experimento apresentavam comprimento total variando entre 26,0 e $32,0 \mathrm{~cm}$ e peso total, entre 155,0 e 378, $0 \mathrm{~g}$.

As 29 fêmeas selecionadas para indução da reprodução apresentavam ovócitos cinzentos ou cor de vinho com diâmetros variando de 1650 a $2150 \mu \mathrm{m}$, valores superiores aos encontrados para Brycon insignis e Brycon cephalus por Andrade-Talmelli (1997) e Romagosa (1998), respectivamente. Quanto à vesícula germinativa (núcleo), em 35\% dos ovócitos ocupava a posição central e em $65 \%$ deles apresentava-se ligeiramente excêntrica, indicando que o ovário se encontrava na fase adiantada de maturação, e, segundo Lam (1982), após esta fase a vesícula germinativa migra para um dos pólos do ovócito e se rompe momentos antes da ovulação. Isto foi observado em fêmeas que responderam ao tratamento hormonal. Resultados semelhantes foram observados por Godinho et al. (1984b) para Prochilodus scrofa, Belmont (1994) para Brycon orbygnianus e Andrade-Talmelli (1997) para Brycon insignis.

Dentre as 14 fêmeas de Brycon opalinus, aquelas que responderam positivamente ao tratamento hormonal, aquelas que apresentaram alta taxa de fertilização mostraram distribuição de freqüência porcentual dos diâmetros dos ovócitos com moda em $1900 \mu \mathrm{m}$ (Figura 1), bem superior à de $1250 \mu \mathrm{m}$,

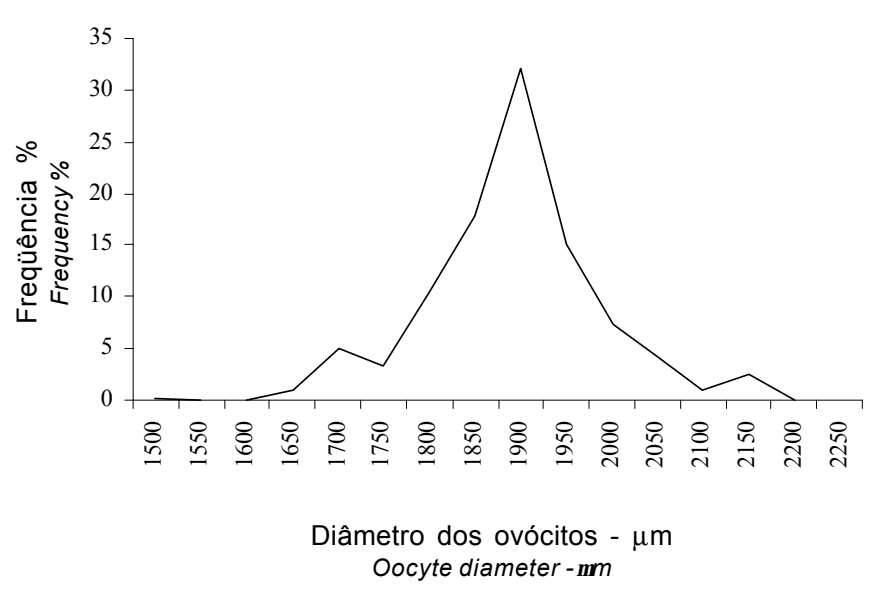

Figura 1 - Distribuição de freqüência porcentual dos diâmetros dos ovócitos de Brycon opalinus (respostas positivas).

Figure 1 - Oocyte diameter frequency distribution of Brycon opalinus (positive response). 
observada por Andrade-Talmelli (1997) para Brycon insignis, e diâmetro médio variando de 1810 a $1933 \mu \mathrm{m}$.

Nas 15 fêmeas que não se reproduziram observaram-se distribuições de freqüência porcentual dos diâmetros dos ovócitos com modas iguais ou inferiores a $1850 \mu \mathrm{m}$ ou iguais ou superiores a $2000 \mu \mathrm{m}$ (Figura 2), tendo o diâmetro médio variado de 1841 a $1982 \mu \mathrm{m}$. Após indução hormonal notaram-se ainda, nestas fêmeas, sinais de regressão ovariana, caracterizada pela presença de ovócitos esbranquiçados entre os cinzentos e os cor de vinho. As mesmas constatações foram feitas por Romagosa et al.(1982; 1985a), no curimbatá Prochilodus scrofa e no pacu Piaractus mesopotamicus que não responderam ao tratamento hormonal.

Shehadeh et al. (1973), trabalhando com Mugil cephalus, e Godinho et al. (1984a e 1993), com Mugil liza e Mugil platanus, utilizaram o diâmetro dos ovócitos como critério de seleção de fêmeas para indução da reprodução. No caso do presente trabalho com Brycon opalinus, devido à amplitude de variação observada nos valores de diâmetro médio dos ovócitos, não se pôde utilizar este critério, mas sim o valor modal da distribuição de freqüência porcentual dos diâmetros dos ovócitos, preconizado por Godinho et al. (1982) e Romagosa et al. (1990) e também utilizado por Andrade-Talmelli (1997) em Brycon insignis.

Nas fêmeas dos grupos controle não se observou nenhuma alteração na distribuição de freqüência porcentual dos diâmetros dos ovócitos.

Dos 58 machos, com comprimento total variando entre 25,5 e $28,0 \mathrm{~cm}$ e peso total entre 160,0 e $200,0 \mathrm{~g}$,

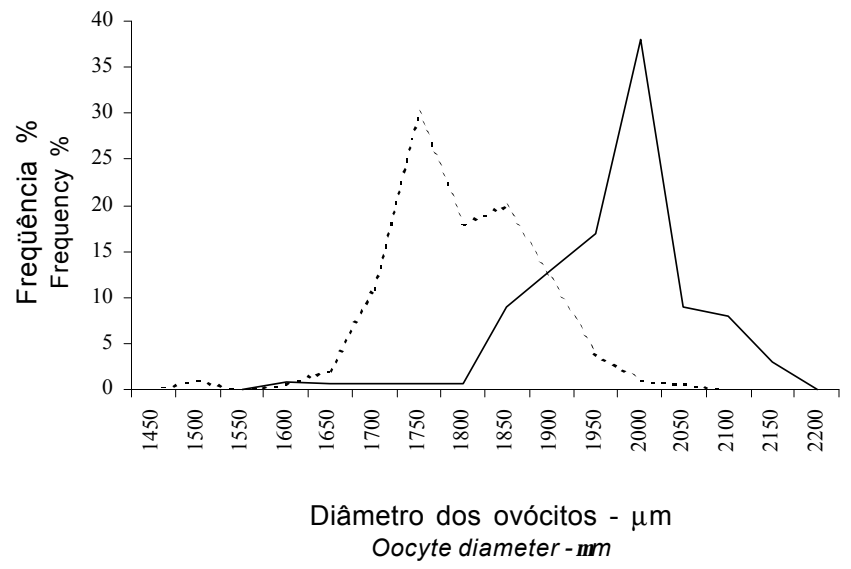

Figura 2 - Distribuições de frequência porcentual dos diâmetros dos ovócitos de Brycon opalinus (respostas negativas)

Figure 2 - Oocyte diameter frequency distributions of Brycon opalinus (negative response) selecionados para receberem o tratamento hormonal, 12 foram examinados quanto à qualidade do sêmen antes de receberem a injeção e apresentaram, em média, $90 \%$ de motilidade espermática, $85 \%$ de células vivas e concentração espermática de $50 \mathrm{x}$ $106 / \mathrm{mm}^{3}$, valores bem próximos aos verificados por Andrade-Talmelli (1997) em Brycon insignis, à exceção da concentração espermática, que, para essa espécie, foi de $24,8 \times 106 / \mathrm{mm}^{3}$. Kavamoto et al. (1986), estudando outras espécies de peixes, obtiveram valores médios de motilidade espermática e coloração diferencial de células vivas inferiores àqueles obtidos para Brycon opalinus. Ainda, os mesmos autores constataram, para Prochilodus scrofa, concentração espermática semelhante à observada para a pirapitinga-do-sul, Brycon opalinus.

Quanto à taxa de fertilização apresentada por Brycon opalinus, neste trabalho, foi alta, ao redor de $90 \%$, e a de eclosão, baixa, aproximadamente $40 \%$, sendo este último valor igual ao observado por Romagosa (1998) para Brycon cephalus e bem próximo (41,8\%) ao encontrado por Andrade-Talmelli (1997) para Brycon insignis.

\section{Conclusões}

Em pirapitinga-do-sul, Brycon opalinus, duas doses de EPS (extrato de pituitária de salmão) nas concentrações de 5 e $10 \mathrm{mg} / \mathrm{kg}$, aplicadas com intervalo de 12 horas, permitiram a indução reprodutiva em $50 \%$ das fêmeas. Uma única dose de $5 \mathrm{UI} / \mathrm{g}$ de hCG foi suficiente para que os machos respondessem positivamente liberando sêmen.

Em pirapitinga-do-sul, distribuição de freqüência porcentual dos diâmetros dos ovócitos com moda de $1900 \mu \mathrm{m}$ indica que as fêmeas estão aptas à reprodução.

A taxa média de fertilização foi de $90 \%$ e a de eclosão, $40 \%$.

\section{Agradecimento}

Aos colegas da CESP e à estagiária Marta Santos Silva, pela importante colaboração durante o desenvolvimento da pesquisa; à MSc Márcia Navarro Cipólli, pela revisão do texto; ao $\mathrm{CNPq}$, pela bolsa concedida; e à Agente de Apoio à Pesquisa Científica e Tecnológica, Maria Regina Guilhermon Rodrigues, pela digitação do texto. 


\section{Literatura Citada}

ANDRADE-TALMELLI, E.F. Indução reprodutiva e ontogenia inicial da piabanha, Brycon insignis, (Steindachner, 1876 (Characiformes, Bryconinae), mantida em confinamento - Vale do Paraíba, SP. São Carlos: Universidade Federal de São Carlos, 1997. 185p. Dissertação (Mestrado em Ecologia e Recursos Naturais) - Universidade Federal de São Carlos, 1997.

BELMONT, R.A.F. Considerações sobre a propagação artificial da piracanjuba, Brycon orbignyanus. In: SEMINÁRIO SOBRE CRIAÇÃO DE ESPÉCIES DO GÊNERO Brycon, Pirassununga. Anais... São Paulo: 1994. p.17-18.

BERNARDINO, G.; ALCANTARA, R.C.G.; ORMANEZI, R. Efeito do LH-RHa na maturação dos ovários de pacu, Colossoma mitrei. In: SÍNTESE DOS TRABALHOS REALIZADOS COM ESPÉCIES DO GÊNERO COLOSSOMA, 1987, Pirassununga. Anais... Pirassununga: CEPTA, 1987. p.13.

BERNARDINO, G.; SENHORINI, J.A.; BOCK, C.L. et al. Propagação artificial do matrinchã Brycon cephalus (Günther, 1869) (Teleostei, Characidae). Boletim Técnico CEPTA, v.6. n.2, p.1-9, 1993.

BLOM, E. A one minute live dead sperm stain by means of eosin-nigrosin. Fertility and Sterility, v.1, n.2, p.176177, 1950.

CASTAGNOLli, N.; CYRINO, J.E.P. Desova induzida do curimbatá Prochilodus scrofa Steindachner, 1881 (Pisces, Prochilodontidae). Ciência e Cultura, v.32, n.9, p.12451253, 1980 .

CASTAGNOLLI, N.; DONALDSON, E.M. Induced ovulation and rearing of the pacu (Colossoma mitrei). Aquaculture, v.25, p.275-279, 1981.

DUMONT-NETO, R.; PELLI, A.; FREITAS, R.O. et al. Reprodução induzida do dourado (Salminus maxillosus, VALENCIENNES, 1849) na Estação de Pesquisa e Desenvolvimento Ambiental de Volta-Grande CEMIG/ EPDA/V.G. Revista Unimar, v.19, n.2, p.439-445, 1997.

ECKMANN. R. Induced reproduction in Brycon cf erythropterus. Aquaculture, v.38, p.379-382, 1984.

FARIA, C.A. Propagação artificial da piabanha (Brycon insignis), na Seção de Hidrobiologia e Aqüicultura de Paraibuna - CESP. In: SEMINÁRIO SOBRE A CRIAÇÃO DE ESPÉCIES DO GÊNERO Brycon, 1., 1994, Pirassununga. Anais... Pirassununga: 1994. p.9-15.

FENERICH, N.A.; GODINHO, H.M.; BARKER, J.M.B. Sobre a ocorrência de ovulação de Rhamdia hilarii, Pimelodus maculatus e Salminus maxillosus em laboratório. Ciência e Cultura, v.26, n.7, p.344, 1974. (Suplemento)

FENERICH-VERANI, N.; GODINHO, H.M.; NARAHARA, M.Y. The size composition of the eggs of curimbatá Prochilodus scrofa, Steindachner, 1881 induced to spawn with human chorionic gonadotropin (HCG). Aquaculture, v.42, p.37-41, 1984.

GODINHO, H.M.; FENERICH, N.A.; NARAHARA, M.Y. et al. Sobre reprodução induzida do pacu Colossoma mitrei (Berg, 1895). Ciência e Cultura, v.29, p.796797, 1977.

GODINHO, H.M.; FENERICH-VERANI, N.; NARAHARA, M.Y. et al. A method for the selection of breeders for induced reproduction of Colossoma mitrei (Berg., 1895).
In: INTERNATIONAL SYMPOSIUM ON REPRODUCTIVE PHYSIOLOGY OF FISH, 1982, Wageningen. Abstract... Wageningen, 1982. p.26.

GODINHO, H.M.; DIAS, E.R.A.; JACOBSEN, O. et al. Reprodução induzida de tainha Mugil liza Val. 1836 da região de Cananéia, SP, Brasil $\left(25^{\circ} 21^{\prime} \mathrm{S}\right)$. SIMPÓSIO BRASILEIRO DE AQÜICULTURA, 3., 1984, São Paulo. Anais... São Paulo: 1984a. p.661-671.

GODINHO, H.M.; ROMAGOSA, E.; CESTAROLLI, M.A. et al. Reprodução induzida de curimbatá Prochilodus scrofa Steind., 1881 sob condições de cultivo experimental. Revista Brasileira de Reprodução Animal, v.8, n.2, p.113-119, 1984b.

GODINHO, H.M.; KAVAMOTO, E.T.; ANDRADETALMELLI, E.F. et al. Induced spawning of the mullet Mugil platanus Günther, 1880, in Cananéia, SP, Brazil. Boletim do Instituto de Pesca, v.20 (único), p.59-66, 1993.

GODINHO, H.P.; GODINHO, A.L. Induced spawning of pacu, Colossoma mitrei (Berg., 1895), by hypophysation with crude carp pituitary extract. Aquaculture, v.55, p.69-73, 1986.

KAVAMOTO, E.T.; FOGLI DA SILVEIRA, W.; GODINHO, H.M. Características seminais do curimbatá Prochilodus scrofa, Steindachner. Boletim do Instituto de Pesca, v.13, n.2, p.45-50, 1986.

LAM, N.A. Applications of endocrinology to fishculture. Canadian Journal of Fisheries and Aquatic Sciences, v.39, p.111-137, 1982.

LEMANOVA, N.A.; SAKUM, O.F. Metodisceskoe posibilie pogor monalnoistimulacji proizvoditielei Karpa pré rannom polucenii licinok. Izvestiya Gosudartvennogo Nauchno - Isseledivatel Skogo Instituta, v.88, p. 323, 1975.

NARAHARA, M.Y.; KAVAMOTO, E.T.; GODINHO, H.M. et al. Pesquisas sobre a criação de espécies do gênero Brycon, no Instituto de Pesca. In: SEMINÁRIO SOBRE A CRIAÇÃO DE ESPÉCIES DO GÊNERO Brycon, 1., 1994, Pirassununga, SP. Anais... Pirassununga: 1994. p.5-6.

ROMAGOSA, E. Desenvolvimento gonadal (morfologia, ultra-estrutura) e indução da reprodução do matrinxã Brycon cephalus (Günther, 1869) em cativeiro Vale do Ribeira, São Paulo. São Carlos: Universidade Federal de São Carlos, 1998. 218p. Tese (Doutorado em Ecologia e Recursos Naturais) - Universidade Federal de São Carlos, 1998.

ROMAGOSA, E.; GODINHO, H.M.; FENERICH-VERANI, N. Observações do processo de regressão do ovário de Prochilodus scrofa Steind., 1881, em tanques de cultivo. Ciência e Cultura, v.34, n.7, p.670, 1982.

ROMAGOSA, E.; PAIVA, P.; CESTAROLli, M.A. et al. Reprodução induzida de pacu Colossoma mitrei, Berg., 1895, mantido em condições de cultivo intensivo. Ciência e Cultura, v.37, n.7, p.849, 1985a.

ROMAGOSA, E.; PAIVA, P.; GODINHO, H.M. et al. Indução da reprodução de pacu, Colossoma mitrei Berg., 1895, em primeira maturação gonadal, mantido sob condições de cultivo intensivo. In: CONGRESSO BRASILEIRO DE ENGENHARIA DE PESCA, Curitiba. Anais... Curitiba: 1985b. p.55.

ROMAGOSA, E.; PAIVA, P.; GODINHO, H.M. Pattern of oocyte diameter frequency distribution in females of the pacu Piractus mesopotamicus (Holmberg, 1887) 
(= Colossoma mitrei Berg, 1895) induced to spawn. Aquaculture, v.86, p.105-110, 1990.

ROMAGOSA, E.; AYROZA, L.M.S.; SANCHES, E.G. et al. Informes preliminares sobre a reprodução induzida do matrinchã, Brycon cephalus. In: SIMPÓSIO BRASILEIRO DE PESQUISAS EM MEDICINA VETERINÁRIA, 1., 1994. Anais... São Paulo: Faculdade de Medicina Veterinária e Zootecnia/Universidade de São Paulo, 1994. p.111.

SATO, Y.; CARDOSO, E.L.; GODINHO, A.L. et al. Hyphophysation parameter of the neotropical fish Prochilodus margravii obtained in hatchery conditions. Revista Brasileira de Biologia, v.56, n.1, p.59-64, 1996a.

SATO, Y.; CARDOSO, E. L.; GODINHO, A.L. et al. Hypophysation of the fish Prochilodus affinis from the Rio São Francisco basin, Brazil. Arquivo Brasileiro de Medicina Veterinária e Zootecnia, v.48, n.1, p.55-62, 1996b.

SATO, Y.; FENERICH-VERANI, N.; GODINHO, H.P. et al. Reprodução induzida do matrinchã (Brycon lundii) Reinhardti, 1877, da bacia do Rio São Francisco. In: SEMINÁRIO REGIONAL DE ECOLOGIA, 8., 1997, São Carlos. Anais... São Carlos: Universidade Federal de São Carlos, 1997. p.353-354.
SHEHADEH, Z.H.; KUO C.M.; MILSEM, K.K. Induced spawning of grey mullet (Mugil cephalus) with fractionated salmon pituitary extract. Journal of Fish Biology, v.5, p.471-478, 1973.

SIMPSON, A.C. The fecundity of the plaice. Fishery Investigation, v.5, n.17, p.1-27, 1951.

TORLONI, C.E.C. Manejo dos recursos pesqueiros nos reservatórios da CESP. São Paulo: CESP-SP, 1992. 16p. (Série Pesquisa e Desenvolvimento, 63)

Von IHERING, R.; AZEVEDO, P. A curimatã dos açudes nordestinos (Prochilodus argenteus). Archivos do Instituto Biológico, v.5, p.143-184, 1934.
Recebido em: 17/05/01

Aceito em: 24/01/02 